\title{
Test of the Universality of Naive-time-reversal-odd Fragmentation Functions
}

\author{
Daniël Boer, ${ }^{1}$ Zhong-Bo Kang, ${ }^{2}$ Werner Vogelsang, ${ }^{3}$ and Feng Yuan ${ }^{4,2}$ \\ ${ }^{1}$ Theory Group, KVI, University of Groningen, Zernikelaan 25, NL-9747 AA Groningen, The Netherlands \\ ${ }^{2}$ RIKEN BNL Research Center, Brookhaven National Laboratory, Upton, NY 11973, USA \\ ${ }^{3}$ Institute for Theoretical Physics, Universität Tübingen, \\ Auf der Morgenstelle 14, D-72076 Tübingen, Germany \\ ${ }^{4}$ Nuclear Science Division, Lawrence Berkeley National Laboratory, Berkeley, CA 94720, USA
}

(Dated: November 23, 2018)

\begin{abstract}
We investigate the "spontaneous" hyperon transverse polarization in $e^{+} e^{-}$annihilation and semiinclusive deep inelastic scattering processes as a test of the universality of the naive-time-reversal-odd transverse momentum dependent fragmentation functions. We find that universality implies definite sign relations among various observables. This provides a unique opportunity to study initial/final state interaction effects in the fragmentation process and test the associated factorization.
\end{abstract}

The universality of parton distribution functions (PDFs) and fragmentation functions (FFs) is a cornerstone of perturbative QCD applications in hadronic physics. It relies on the factorization theorem for the relevant high energy processes, which has been rigorously proven and widely applied in phenomenological studies. In particular, the PDFs depending only on the longitudinal momentum fractions of hadrons carried by the partons have been shown to be universal among different processes [1]. In recent years, however, intensive theoretical investigations have shown that the so-called naivetime-reversal-odd (T-odd) transverse momentum dependent (TMD) PDFs are not universal due to differences in initial/final state interactions. For example, it was found that the difference between the final state interactions in semi-inclusive hadron production in deep inelastic scattering (SIDIS) and initial state interactions in Drell-Yan lepton pair production (DY) in hadronic collisions leads to an opposite sign in the TMD Sivers function for quarks that enters in these two processes $[2[6]$. Measurements of Sivers asymmetries in SIDIS have been carried out experimentally, while those in the DY process are planned. It is of great importance to test this modified universality (sign change), in order to unravel fundamental dynamics in strong interaction physics and the novel aspects of nucleon structure involved in these processes.

On the other hand, their fragmentation counterparts, the T-odd TMD FFs, have been shown to be universal [711. Here, the so-called Collins FFs [12] and polarizing FFs 13 have been the main focus. The test of this universality (i.e., no sign change) is as important as that for the modified universality of the quark Sivers function discussed above. The universality of these FFs involves the same QCD dynamics and arguments for the TMD factorization of the relevant processes.

However, it is difficult to carry out a test of the universality of the Collins FF because of its chiral-odd nature. In order to observe its effect, it has to be coupled with another chiral-odd object. For example, in the SIDIS process, the Collins function couples to the quark transversity distribution to generate a novel single trans- verse spin asymmetry; or in the $e^{+} e^{-}$annihilation process, two Collins functions couple together and lead to an asymmetric azimuthal angular correlation of di-hadron production. The issue is that the sign of these chiral-odd objects is not easy to determine. For example, one can change the sign of all functions involved without altering the actual physical observables. Thus, in order to test universality, one needs to be able to determine the relative signs, which will necessarily involve additional chiral-odd functions and therefore further observables.

In this paper, we investigate a test of the universality of the T-odd TMD FFs by studying hyperon polarization in the SIDIS and $e^{+} e^{-}$annihilation processes. The relevant T-odd FFs describe the transverse polarization of the hyperon, typically a $\Lambda$, correlated with its transverse momentum relative to the fragmenting quark jet [13 16$]$. The universality of this fragmentation function was first demonstrated in a model calculation in Ref. [7], and subsequently using model-independent arguments [8]. It is a chiral-even function, and will couple to chiral-even functions in hadronic processes, such as the spin-averaged quark PDFs in SIDIS and the spin-averaged FFs in $e^{+} e^{-}$ processes. Because these functions are well determined and most importantly all positive, it will be possible to unambiguously measure the sign of the T-odd FFs and test their universality.

The TMD quark FFs can be defined through the following correlator:

$$
\begin{aligned}
\Delta\left(z_{h}, p_{\perp}\right)= & \frac{1}{z_{h}} \int \frac{d y^{-} d^{2} y_{\perp}}{(2 \pi)^{3}} e^{i k \cdot y}\left\langle 0\left|\mathcal{L}_{y} \psi(y)\right| P_{h} S_{\perp} X\right\rangle \\
& \times\left.\left\langle P_{h} S_{\perp} X\left|\bar{\psi}(0) \mathcal{L}_{0}^{\dagger}\right| 0\right\rangle\right|_{y^{+}=0}
\end{aligned}
$$

where $P_{h}$ is the momentum of the final state hadron with spin $S_{\perp}$, which has a transverse component $p_{\perp}$ relative to the momentum $k$ of the fragmenting quark. We choose the hadron to move along the $+z$ direction, and define the light-cone components $p^{ \pm}=\left(p^{0} \pm p^{z}\right) / \sqrt{2}$. For convenience, we define two light-like vectors: $\bar{n}^{\mu}=\delta^{\mu+}$ and $n^{\mu}=\delta^{\mu-}$. The momentum fraction $z_{h}=P_{h}^{+} / k^{+}$, and 
$\vec{k}_{\perp}=-\vec{p}_{\perp} / z_{h}$. The correlator can then be expanded as

$$
\begin{aligned}
\Delta\left(z_{h}, p_{\perp}\right)= & \frac{1}{2}\left[D\left(z_{h}, p_{\perp}^{2}\right) \not{h}+\frac{1}{M_{h}} D_{1 T}^{\perp}\left(z_{h}, p_{\perp}^{2}\right)\right. \\
& \left.\times \epsilon^{\mu \nu \rho \sigma} \gamma_{\mu} \bar{n}_{\nu} p_{\perp \rho} S_{\perp \sigma}+\cdots\right]
\end{aligned}
$$

where we have only kept the FFs of relevance here: the spin-averaged $D\left(z_{h}, p_{\perp}^{2}\right)$, and the spin-dependent T-odd one $D_{1 T}^{\perp}\left(z_{h}, p_{\perp}^{2}\right)$, usually called "polarizing FF". The latter leads to single-transverse-spin asymmetries in hyperon production in various processes 14 16]. It offers an explanation for the "spontaneous" hyperon polarization observed in hadron-hadron collisions many years ago [17].

In Eq. (11), the gauge link is defined as $\mathcal{L}_{y}=$ $\mathcal{P} \exp \left(i g \int_{0}^{\infty} d \lambda v \cdot A(y+\lambda v)\right)$ with $v$ satisfying $v^{-} \gg v^{+}$ and $v^{2} \neq 0$ to regulate the light-cone singularity [4]. The gauge link results from the initial/final state interactions.

The polarizing FF cannot be calculated from first principles, but it can be computed in perturbation theory for large $p_{\perp}$, yielding its so-called perturbative tail. Since this has not been given elsewhere we present it here:

$$
\begin{aligned}
D_{1 T}^{\perp}\left(z_{h}, p_{\perp}^{2}\right)= & \frac{\alpha_{s}}{2 \pi^{2}} \frac{M_{h}}{\left(p_{\perp}^{2}\right)^{2}} \int \frac{d z}{z}[A(z) \\
& \left.+\delta(\hat{z}-1) \hat{T}(z) C_{F}\left(\ln \frac{\hat{\zeta}^{2}}{p_{\perp}^{2}}-1\right)\right],
\end{aligned}
$$

where $\hat{z}=z_{h} / z, \hat{\zeta}^{2}=\left(v \cdot P_{h}\right)^{2} / v^{2}$, and $A$ is given by:

$$
\begin{aligned}
A(z)= & C_{F}\left[-\left(1+\hat{z}^{2}\right) z \frac{\partial \hat{T}(z)}{\partial z}-\hat{T}(z) \frac{2 \hat{z}^{3}-3 \hat{z}^{2}-1}{(1-\hat{z})_{+}}\right] \\
& +\int \frac{d z_{1}}{z_{1}^{2}} \mathrm{PV}\left(\frac{1}{\frac{1}{z}-\frac{1}{z_{1}}}\right) \hat{T}_{F}\left(z, z_{1}\right) \\
& \times\left[C_{F}\left(\frac{z}{z_{1}}-\frac{z_{h}}{z_{1}}+\frac{z_{h}}{z}-\frac{z_{h}^{2}}{z z_{1}}-2\right)\right. \\
& \left.+\frac{C_{A}}{2} \frac{\left(z z_{h}+z_{1} z_{h}-2 z z_{1}\right)\left(z z_{1}+z_{h}^{2}\right)}{\left(z-z_{1}\right)\left(z_{1}-z_{h}\right) z^{2}}\right]
\end{aligned}
$$

We note that the first two terms in $A$ have the same structure as the soft-pole contributions to the perturbative tail of the Sivers PDF [18]. In Eqs. (3), (44) we have

$$
\begin{aligned}
\hat{T}(z)= & z^{2} \int \frac{d y^{-}}{2 \pi} e^{i k^{+} y^{-}} \frac{1}{2}\left\{\operatorname { T r } \not h \langle 0 | \epsilon ^ { n \overline { n } S _ { \perp } \alpha } \left[i D_{\perp \alpha}\right.\right. \\
& \left.+\int_{y^{-}}^{+\infty} d \xi^{-} g F_{\alpha}^{+}\left(\xi^{-}\right)\right] \psi\left(y^{-}\right)\left|P_{h} S_{\perp} X\right\rangle \\
& \left.\times\left\langle P_{h} S_{\perp} X|\bar{\psi}(0)| 0\right\rangle+\text { h.c. }\right\},
\end{aligned}
$$

with $D_{\perp}^{\alpha}=\partial_{\perp}^{\alpha}-i g A_{\perp}^{\alpha}$ the covariant derivative, $F^{\alpha \beta}$ the field strength tensor, and

$$
\begin{aligned}
\hat{T}_{F}\left(z_{1}, z_{2}\right) & =z_{1} z_{2} \int \frac{d y^{-}}{2 \pi} \frac{d \xi^{-}}{2 \pi} e^{i k_{2}^{+} y^{-}} e^{i k_{g}^{+} \xi^{-}} \\
& \times \frac{1}{2}\left\{\operatorname{Tr} \not h\left\langle 0\left|\epsilon^{n \bar{n} S_{\perp} \alpha} g F^{+}{ }_{\alpha}\left(\xi^{-}\right) \psi\left(y^{-}\right)\right| P_{h} S_{\perp} X\right\rangle\right. \\
& \left.\times\left\langle P_{h} S_{\perp} X|\bar{\psi}(0)| 0\right\rangle+\text { h.c. }\right\}
\end{aligned}
$$

where $k_{g}^{+}=k_{1}^{+}-k_{2}^{+}$with $k_{1}^{+}=P_{h}^{+} / z_{1}$ and $k_{2}^{+}=P_{h}^{+} / z_{2}$. The twist-three correlator $\hat{T}$ is related to $D_{1 T}^{\perp}$ as follows:

$$
\hat{T}(z)=\int d^{2} p_{\perp} \frac{\left|\vec{p}_{\perp}\right|^{2}}{M_{h}} D_{1 T}^{\perp}\left(z, p_{\perp}^{2}\right) .
$$

In the calculation of the perturbative tail we have verified explicitly the gauge-link independence of the result, which is equivalent to establishing the universality of $D_{1 T}^{\perp}$ in the perturbative region. Arguments for its general universality have been given based on the vanishing of the so-called gluonic pole matrix elements in the fragmentation function [8, 10]. The universality is based on the property [8] $\left.\hat{T}_{F}\left(z_{1}, z_{2}\right)\right|_{z_{1} \geq z_{2}}=0$, or equivalently, $\left.\hat{T}_{F}\left(z_{1}, z_{2}\right)\right|_{k_{g}^{+} \leq 0}=0$. Intuitively, this means that the parton momentum entering hadronic matrix elements in the fragmentation function has to be positive.

This property also turns out to be essential for the universality of the perturbative tail, and our calculation hence provides a consistency check for the arguments in Refs. [8, 10]. At large transverse momentum, a hard

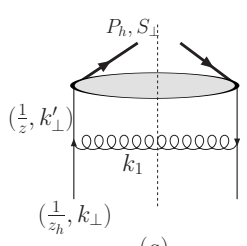

(a)

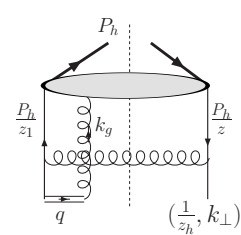

(b)

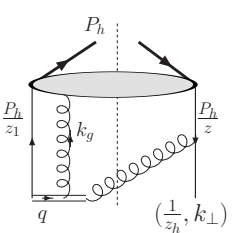

(c)
FIG. 1: Sample Feynman diagrams contributing to the naive T-odd spin dependent FF at large transverse momentum. The double lines in $(b, c)$ represent the eikonal propagators from the gauge link expansion of the fragmentation function definition. The pole contributions from the eikonal propagators vanish.

gluon has to be radiated, as shown in Fig. 1 (a) is a typical quark splitting contribution to the fragmentation function; (b) and (c) are sample diagrams with gauge link contributions. The gauge link represents the initial/final state interactions in the associated processes. In the calculation of the gauge link, eikonal propagators of the form $1 /\left(q^{+} \pm i \epsilon\right)$ arise, where the $i \epsilon$ prescription depends on the gauge link direction (initial or final state interaction). As is well-known, this is responsible for the nontrivial universality properties of the T-odd Sivers functions. For the FFs, however, the pole contributions from the gauge link vanish, such that the T-odd FFs are universal among different processes [7 9]. Specifically, in the diagram in Fig. 1(b) the pole contribution (soft pole) from the eikonal propagator leads to a deltafunction $\delta\left(q^{+}\right)=\delta\left(k_{g}^{+}\right)$, where the associated twist-three correlation function $\left.\hat{T}_{F}\left(z, z_{1}\right)\right|_{k_{g}^{+}=0}$ vanishes [8]. Similarly, in Fig. 1(c), the pole contribution (hard pole) leads to $\delta\left(q^{+}\right)=\delta\left(k_{g}^{+}+k_{1}^{+}\right)$where $k_{1}$ is the momentum of the radiated gluon. Since $k_{1}^{+}>0$ by kinematics which results in $k_{g}^{+}<0$, the associated twist-three function 
vanishes again, $\left.\hat{T}_{F}\left(z, z_{1}\right)\right|_{k_{g}^{+}<0}=0$. All other diagrams can be analyzed accordingly. Therefore, there is no contribution from either the soft or hard poles associated with the eikonal propagator in the gauge link expansion. The conclusion is that the T-odd fragmentation function calculated from these perturbative diagrams does not depend on the gauge link direction, and hence is universal. We emphasize, however, that the gauge link contributions are important for guaranteeing the gauge invariance of the fragmentation function.

We now discuss the possibility to test the universality of $D_{1 T}^{\perp}$ in experiment. Here we consider the $\Lambda$ hyperon as an example. In our calculations, we focus on the polarization of $\Lambda$ only, but extension to $\bar{\Lambda}$ and $\Lambda+\bar{\Lambda}$ can follow similarly. As it turns out, the comparison of the asymmetries induced by $D_{1 T}^{\perp}$ in SIDIS $\left(\ell p \rightarrow \ell^{\prime} \Lambda^{\uparrow} X\right)$ [13, 16] and $e^{+} e^{-}$annihilation $\left(e^{+} e^{-} \rightarrow \Lambda^{\uparrow}\right.$ jet $\left.X\right)[14$ is not straightforward. The problem is the possibility of cancellation among different flavors, which depends on the unknown magnitude of $S U(3)$ breaking and which can lead to sign reversal of the asymmetries. This also applies to another test of universality of $D_{1 T}^{\perp}$ proposed in Ref. [19]. Here we will show that detection of the $\Lambda$ in coincidence with a light hadron $h$ in $e^{+} e^{-}$annihilation alleviates the situation significantly and offers a robust test of the universality, to be performed for instance with SIDIS at COMPASS, HERMES, Jefferson Lab, or a future electron-ion collider (EIC) and with $e^{+} e^{-}$at BELLE.

The hyperon (transverse) polarization is defined as $P_{T}^{\Lambda}=d \Delta \sigma\left(S_{\perp}\right) / d \sigma$ with $d \Delta \sigma\left(S_{\perp}\right)=$ $\frac{1}{2}\left[d \sigma\left(S_{\perp}\right)-d \sigma\left(-S_{\perp}\right)\right]$ and $d \sigma=\frac{1}{2}\left[d \sigma\left(S_{\perp}\right)+d \sigma\left(-S_{\perp}\right)\right]$. The differential cross section for a correlation of a $\Lambda$ and a light hadron in $e^{+} e^{-}, e^{-}(\ell)+e^{+}\left(\ell^{\prime}\right) \rightarrow \gamma^{*}(q) \rightarrow$ $\Lambda^{\uparrow}\left(P_{\Lambda}, S_{\perp}\right)+h\left(P_{h}\right)+X$ can be written as

$$
\begin{aligned}
\frac{d \Delta \sigma\left(S_{\perp}\right)}{d z_{\Lambda} d z_{h} d y d^{2} q_{\perp}}= & \sigma_{0} \epsilon^{\alpha \beta} S_{\perp \alpha} q_{\perp \beta} \frac{1}{q_{\perp}^{2}} z_{\Lambda}^{2} z_{h}^{2} \int_{\perp} \frac{-z_{\Lambda} \vec{k}_{\Lambda \perp} \cdot \vec{q}_{\perp}}{M_{\Lambda}} \\
& \times D_{1 T}^{\perp}\left(z_{\Lambda}, z_{\Lambda}^{2} k_{\Lambda \perp}^{2}\right) D\left(z_{h}, z_{h} k_{h \perp}^{2}\right) \\
& \times\left(S\left(\lambda_{\perp}\right)\right)^{-1} H\left(Q^{2}\right),
\end{aligned}
$$

where $Q^{2}=q^{2}=\left(\ell+\ell^{\prime}\right)^{2}, z_{i}=2 P_{i} \cdot q / Q^{2}$ with $i=\Lambda, h$, $y=P_{h} \cdot \ell / P_{h} \cdot q, \sigma_{0}=N_{c} 4 \pi \alpha_{e m}^{2}\left(1 / 2-y+y^{2}\right) / Q^{2}$ with $\alpha_{e m}$ the electromagnetic coupling, and the integral symbol $\int_{\perp} \equiv \int d^{2} k_{\Lambda \perp} d^{2} k_{h \perp} d^{2} \lambda_{\perp} \delta^{2}\left(\vec{k}_{\Lambda \perp}+\vec{k}_{h \perp}+\vec{\lambda}_{\perp}-\vec{q}_{\perp}\right) . q_{\perp}$ is related to the "transverse" component of the virtual photon momentum defined as $q_{t}^{\mu}=q^{\mu}-\frac{P_{h} \cdot q}{P_{h} \cdot P_{\Lambda}} P_{\Lambda}^{\mu}-\frac{P_{\Lambda} \cdot q}{P_{\Lambda} \cdot P_{h}} P_{h}^{\mu}$, with $q_{\perp}^{2}=-q_{t}^{\mu} q_{t \mu}$.

Similarly, the differential cross section for $\Lambda$ production in SIDIS, $e(\ell)+p(P) \rightarrow e\left(\ell^{\prime}\right)+\Lambda^{\uparrow}\left(P_{\Lambda}, S_{\perp}\right)+X$, reads

$$
\begin{aligned}
\frac{d \Delta \sigma\left(S_{\perp}\right)}{d x_{B} d y d z_{\Lambda} d^{2} P_{\Lambda \perp}}= & \sigma_{0}^{D I S} \epsilon^{\alpha \beta} S_{\perp \alpha} P_{\Lambda \perp \beta} \frac{1}{P_{\Lambda \perp}^{2}} \int_{\perp} \frac{\vec{p}_{\perp} \cdot \vec{P}_{\Lambda \perp}}{M_{\Lambda}} \\
& \times q\left(x_{B}, k_{\perp}\right) D_{1 T}^{\perp}\left(z_{\Lambda}, p_{\perp}\right) \\
& \times\left(S\left(\lambda_{\perp}\right)\right)^{-1} H\left(Q^{2}\right)
\end{aligned}
$$

where $Q^{2}=-q^{2}=-\left(\ell^{\prime}-\ell\right)^{2}, x_{B}=Q^{2} / 2 P \cdot q, z_{\Lambda}=P$. $P_{\Lambda} / P \cdot q, y=P \cdot q / P \cdot \ell, \sigma_{0}^{D I S}=4 \pi \alpha_{\mathrm{em}}^{2}(1 / y-1+y / 2) / Q^{2}$, $q\left(x_{B}, k_{\perp}\right)$ is the spin-averaged quark distribution, and $\int_{\perp} \equiv \int d^{2} k_{\perp} d^{2} p_{\perp} d^{2} \lambda_{\perp} \delta^{2}\left(z_{\Lambda} \vec{k}_{\perp}+\vec{p}_{\perp}+\vec{\lambda}_{\perp}-\vec{P}_{\Lambda \perp}\right)$. In both Eqs. (8) and (9), $S\left(\lambda_{\perp}\right)$ and $H\left(Q^{2}\right)$ denote soft and hard factors, respectively. We have ignored contributions from chiral-odd functions 20 22 that lead to different azimuthal dependences and therefore can be either distinguished or averaged out.

We will now estimate the $\Lambda$ polarization $P_{T}^{\Lambda}$ in both processes. We will assume the lowest order results for the soft and hard factors in both Eqs. (8) and (9): $\left(S\left(\lambda_{\perp}\right)\right)^{-1}=\delta^{2}\left(\lambda_{\perp}\right)$ and $H\left(Q^{2}\right)=1$. As in [15, 16], we keep the transverse momentum dependence only in $D_{1 T}^{\perp}$ and drop it in the spin-averaged PDFs and FFs. We choose the initial hadron in SIDIS or the final hadron in $e^{+} e^{-}$along the $-z$ direction, while $x$ is along the transverse momentum of the $\Lambda$. Thus the transverse polarization is measured along $+y$. The azimuthal angle dependence will be $d \sigma\left(S_{\perp}\right) \propto\left|S_{\perp}\right| P_{\Lambda \perp} \sin \phi$, where $\phi$ is the angle between $S_{\perp}$ and $P_{\Lambda \perp}$, and $\phi=\pi / 2$ in our frame.

We adopt the parametrizations of $D_{1 T}^{\perp}$ obtained in [16],

$$
D_{1 T}^{\perp q}\left(z_{h}, k_{\perp}\right)=f^{\Delta}\left(z_{h}, k_{\perp}\right) D_{\Lambda / q}\left(z_{s}, k_{\perp}\right),
$$

where $D_{1 T}^{\perp q}$ is proportional to the spin-averaged $\Lambda$ FFs $D_{\Lambda / q}\left(z_{h}, k_{\perp}\right)$ with the proportionality coefficient $f^{\Delta}\left(z_{h}, k_{\perp}\right)$ a function fitted to the available experimental data. The $k_{\perp}$-dependence has been assumed to be Gaussian in both functions. Several spin-averaged FFs have been considered, such as the $S U(3)$ flavor symmetric 23. or broken 24] ones. Since both types of parametrizations fit the available data, a strong dependence on $S U(3)$ breaking would jeopardize the test of universality in the case of small asymmetries. In Fig. 2, we show the $\Lambda$ polarization as a function of $z_{\Lambda}$ in both semi-inclusive DIS $e p \rightarrow e^{\prime}+\Lambda^{\uparrow}+X$ (left) and $e^{+} e^{-}$annihilation $e^{+} e^{-} \rightarrow \pi^{ \pm}+\Lambda^{\uparrow}+X$ (right). For the latter, we have used the pion fragmentation functions of Ref. 25]. Our numerical results show that the polarizations are sizable, and have the same sign in both processes, irrespective of the size of the $S U(3)$ breaking. In both SIDIS and $e^{+} e^{-}$annihilation, up quarks dominate the scattering. Since $D_{1 T}^{\perp u}$ is negative in the models we are using, the negative sign of $P_{T}^{\Lambda}$ in SIDIS follows immediately. For $e^{+} e^{-} \rightarrow \pi^{-}+\Lambda^{\uparrow}+X$, the dominant mechanism is upquark fragmentation to the $\Lambda$ and $\bar{u}$-fragmentation to the $\pi^{-}$, again resulting in a negative asymmetry. For positive pions, there is some competition among the various flavor combinations, and there is a larger contribution by $D_{1 T}^{\perp d}$. Since the latter is negative as well, the asymmetry remains negative. Therefore universality implies equality of the signs of the asymmetries in the two processes. Fig. [3] shows that the process $e^{+} e^{-} \rightarrow$ jet $+\Lambda^{\uparrow}+X$, on the other hand, is much more sensitive to flavor cancellation effects, and its sign relation to the SIDIS polarization 


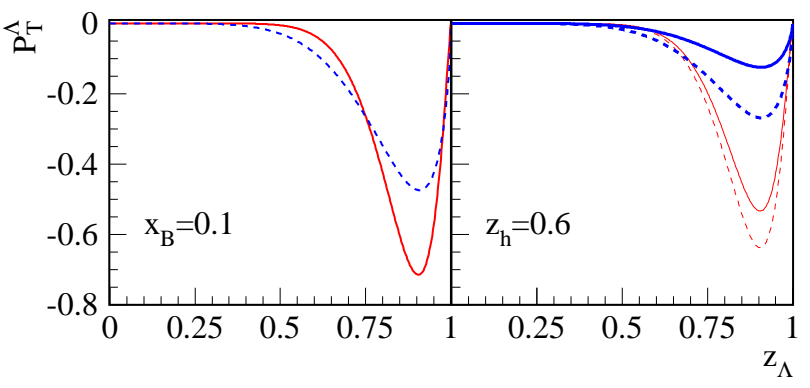

FIG. 2: Universality predictions of transverse $\Lambda$ polarization $P_{T}^{\Lambda}$ as function of $z_{\Lambda}$. Left: SIDIS ep $\rightarrow e^{\prime}+\Lambda^{\uparrow}+X$ at $\left\langle Q^{2}\right\rangle \sim$ $10 \mathrm{GeV}^{2}$ and $x_{B} \sim 0.1$. We have integrated over $P_{\Lambda \perp} \leq$ $3 \mathrm{GeV}$. The solid (dashed) line is for the $S U(3)$-symmetric (broken) spin-averaged $\Lambda$ FFs. Right: $e^{+} e^{-} \rightarrow \pi^{ \pm}+\Lambda^{\uparrow}+X$ at $\left\langle Q^{2}\right\rangle \sim 100 \mathrm{GeV}^{2}$ and $z_{h} \sim 0.6$. Thin (thick) lines are for the $S U(3)$-symmetric (broken) spin-averaged $\Lambda$ FFs. The solid (dashed) lines are for $\pi^{+}\left(\pi^{-}\right)$.

would not yield a robust test. Also shown in Fig. 3 is $\Lambda+$ kaon production in $e^{+} e^{-}$annihilation. We observe that $K^{-}$production gives results very similar to those for $\pi^{-}$, so that $\Lambda$ plus negatively charged hadrons would likely do equally well. For positive kaons, we find that the $\Lambda$ polarization flips sign, as a result of the relatively bigger contribution from the $s \rightarrow \Lambda$ fragmentation function, which is expected positive. It will thus be possible to unambiguously measure the signs of the T-odd FFs. With precise experimental data one could further perform a global analysis to see if a universal set of polarizing FFs could be obtained. We note that the opposite signs of the $u / d$ and $s$ quark polarizing FFs used here arise naturally to ensure that $\bar{\Lambda}$ polarization in $p p$ collisions is consistent with zero [15]. Although the uncertainties in the polarizing FFs are rather large in general, as also witnessed by the fact that the calculated polarization may exceed unity, the principle of fixing the sign by selecting $u / d$ or $s$ quark dominanted processes is expected to be robust.

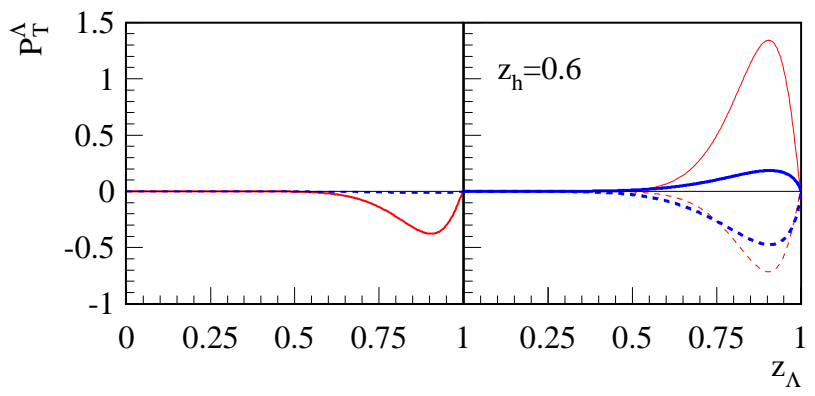

FIG. 3: $\Lambda$ hyperon (transverse) polarization $P_{T}^{\Lambda}$ as function of $z_{\Lambda}$ at $\left\langle Q^{2}\right\rangle \sim 100 \mathrm{GeV}^{2}$. Left: $e^{+} e^{-} \rightarrow$ jet $+\Lambda^{\uparrow}+X$. The solid (dashed) line is for the $S U(3)$-symmetric (broken) spinaveraged $\Lambda$ FFs. Right: $e^{+} e^{-} \rightarrow K^{ \pm}+\Lambda^{\uparrow}+X$ at $z_{h} \sim 0.6$. Thin (thick) lines are for the $S U(3)$-symmetric (broken) spinaveraged FFs. The solid (dashed) lines are for the $K^{+}\left(K^{-}\right)$.

In summary, we have investigated the universal- ity of the T-odd spin-dependent FFs using hyperon (transverse) polarization in $e^{+} e^{-}$annihilation and semiinclusive DIS processes. Definite signs for the polarization are predicted based on the current knowledge of the polarizing fragmentation functions. Despite the large uncertainties in these functions, the obtained sign relations among the measured polarizations constitute a robust test of universality, in the sense that they are necessary conditions for universality to hold. We hope that this test can be carried out in the near future. It would provide an important confirmation of our current understanding of novel single spin asymmetries in high energy hadronic reactions and the associated QCD dynamics.

This work was supported in part by the U.S. Department of Energy under grant number DE-AC02$05 \mathrm{CH} 11231$ (FY) and contract number DE-AC0298CH10886 (ZK, FY and WV).

[1] J. C. Collins et al., Adv. Ser. Direct. High Energy Phys. 5, 1 (1988); G. C. Nayak, J. W. Qiu and G. F. Sterman, Phys. Rev. D 72, 114012 (2005).

[2] S. J. Brodsky et al., Phys. Lett. B 530, 99 (2002); Nucl. Phys. B 642, 344 (2002).

[3] J. C. Collins, Phys. Lett. B 536, 43 (2002).

[4] X. Ji and F. Yuan, Phys. Lett. B 543, 66 (2002); A. V. Belitsky et al., Nucl. Phys. B 656, 165 (2003).

[5] D. Boer et al., Nucl. Phys. B 667, 201 (2003).

[6] D. W. Sivers, Phys. Rev. D 41, 83 (1990); Phys. Rev. D 43, 261 (1991).

[7] A. Metz, Phys. Lett. B 549, 139 (2002); J. C. Collins and A. Metz, Phys. Rev. Lett. 93, 252001 (2004).

[8] S. Meissner and A. Metz, Phys. Rev. Lett. 102, 172003 (2009).

[9] F. Yuan, Phys. Rev. Lett. 100, 032003 (2008); Phys. Rev. D 77, 074019 (2008).

[10] L. P. Gamberg et al., Phys. Rev. D 77, 114026 (2008).

[11] F. Yuan and J. Zhou, Phys. Rev. Lett. 103, 052001 (2009).

[12] J. C. Collins, Nucl. Phys. B 396, 161 (1993).

[13] P. J. Mulders and R. D. Tangerman, Nucl. Phys. B 461, 197 (1996) [Erratum-ibid. B 484, 538 (1997)].

[14] D. Boer et al., Nucl. Phys. B 504, 345 (1997).

[15] M. Anselmino et al., Phys. Rev. D 63, 054029 (2001).

[16] M. Anselmino et al., Phys. Rev. D 65, 114014 (2002); D. Boer et al., Phys. Lett. B 671, 91 (2009).

[17] G. Bunce et al., Phys. Rev. Lett. 36, 1113 (1976); K. J. Heller et al., Phys. Rev. Lett. 41, 607 (1978) [Erratum-ibid. 45, 1043 (1980)].

[18] X. Ji et al., Phys. Rev. Lett. 97, 082002 (2006); Phys. Rev. D 73, 094017 (2006); Phys. Lett. B 638, 178 (2006).

[19] D. Boer et al., Phys. Lett. B 659, 127 (2008).

[20] Y. Kanazawa and Y. Koike, arXiv:hep-ph/0012005 Phys. Rev. D 64, 034019 (2001).

[21] J. Zhou et al., Phys. Rev. D 78, 114008 (2008).

[22] D. Boer and P. J. Mulders, Phys. Rev. D 57, 5780 (1998).

[23] D. de Florian et al., Phys. Rev. D 57, 5811 (1998).

[24] D. Indumathi et al., Phys. Rev. D 58, 094014 (1998).

[25] D. de Florian et al., Phys. Rev. D 75, 114010 (2007). 